\title{
ML4UL: Uma Extensão ao Padrão IMS LD para Modelagem de Sistemas Móveis e Ubíquos para Aulas de Campo
}

\author{
Edgar Marçal, Rossana M. C. Andrade, Windson Viana \\ Mestrado e Doutorado em Ciência da Computação - Universidade Federal do Ceará \\ (UFC) - Fortaleza, CE - Brasil \\ edgarevirtual.ufc.br, rossana@lia.ufc.br, windsonevirtual.ufc.br
}

\begin{abstract}
Field trips are used by teachers as educational practices to motivate student learning. Research has been undertaken to expand the benefits provided by these activities through mobile and ubiquitous systems. An approach to the construction of this type of software is to use model-driven development through modeling languages. In the area of teaching and learning systems, the educational modeling language that stands out is IMS LD. However, this model has limitations when considering mobile and ubiquitous technologies applied in field trips. Thus, this paper proposes an extension to the IMS LD specification for mobile and ubiquitous systems applied to field trips. A scenario and an application that demonstrate how the extension works are also presented.
\end{abstract}

Resumo. As aulas de campo são utilizadas pelos professores como práticas educativas para motivar o aprendizado dos alunos. Pesquisas têm sido desenvolvidas no sentido de ampliar os beneficios proporcionados por essas atividades através de sistemas móveis e ubíquos. Uma abordagem para a construção desse tipo de software é a utilização do desenvolvimento dirigido por modelos através de linguagens de modelagem. Na área de sistemas de ensino e aprendizagem, o padrão de modelagem que se destaca é o IMS LD. Porém, ao se considerar as tecnologias móveis e ubíquas aplicadas em aulas de campo, esse modelo apresenta limitações. Assim, esse artigo propõe uma extensão ao IMS LD para especificação de sistemas móveis e ubíquos aplicados a aulas de campo. Um cenário de uso e um exemplo de aplicação que demonstram o funcionamento da extensão são também apresentados.

\section{Introdução}

A aula de campo é uma metodologia de ensino que proporciona diferentes benefícios à aprendizagem, caracterizando-se como uma ação motivadora que permite aos alunos aprenderem na prática o que foi lecionado em sala de aula [Shakil et al., 2011]. Essas práticas de campo são utilizadas tanto no ensino superior (e.g., em viagens para afloramentos geológicos) quanto no ensino básico (e.g., em excursões para museus).

Ao integrar nessas atividades em campo as tecnologias móveis e ubíquas, novos recursos podem ser adicionados aos processos de ensino e aprendizagem [Marçal et al., 2013; Wu et al., 2013; Chiang et al., 2014]. Dentre esses recursos, podemos citar: identificação do contexto do aluno para a recomendação de conteúdos e exercícios de acordo com a situação dele naquele momento; registro das ações e preferências dos aprendizes para recomendações futuras em atividades similares; utilização de sensores (embutidos ou acoplados ao dispositivo móvel) para coleta e registro de informações 
relevantes à aprendizagem em campo; e, comunicação entre alunos e professores através de redes sem fio para esclarecimento de dúvidas ou compartilhamento de informações.

De acordo com [Sharples e Roschelle, 2010], esse novo estilo de aplicação educacional se encontra na terceira geração do paradigma conhecido como mobile learning. Essas novas aplicações são marcadas pela sua ubiquidade e a elas se aplicam o termo ubiquitous learning ou u-learning. Na terceira geração, as características dos sistemas ubíquos são utilizadas para ampliar os recursos das práticas educativas.

O desenvolvimento de sistemas móveis e ubíquos impõe desafios como o tratamento de forma transparente da heterogeneidade dos dispositivos, a ausência de infraestrutura de comunicação fixa, a dinamicidade do ambiente e o suporte à mobilidade dos usuários. Considerando essa complexidade, a construção desse tipo de sistema por professores ou especialistas de domínios sem conhecimentos de programação torna-se uma tarefa inviável. Uma alternativa para abstrair os aspectos complexos de codificação no desenvolvimento de sistemas é a utilização de linguagens específicas de domínio (em inglês Domain-Specific Language - DSL). Através das DSLs, uma aplicação pode ser modelada em uma linguagem de alto nível e, posteriormente, ter o aplicativo executável gerado [Brambilla et al., 2012].

Nesse sentindo, o IMS Learning Design (IMS LD) apresenta-se como um padrão adotado por várias instituições de ensino e baseado em uma linguagem capaz de modelar sistemas de e-learning com diferentes abordagens pedagógicas [Koper e Miao, 2007]. Porém, ao se considerar os sistemas móveis e ubíquos para aulas de campo, o padrão apresenta limitações, em virtude principalmente da ausência de conexão com a Internet nas aulas de campo e da utilização de tecnologias móveis e ubíquas.

Assim, esse artigo propõe a ML4UL (Modeling Language for Ubiquitous Learning), uma extensão à linguagem de modelagem do IMS LD para especificação de sistemas móveis e ubíquos aplicados a aulas de campo. Ao longo do artigo, serão mostrados: um cenário de uso das aplicações, as limitações identificadas na IMS LD e os novos elementos propostos que compõem a extensão. Posteriormente, será apresentado um exemplo que demonstra a especificação de um sistema móvel e ubíquo usando os elementos da ML4UL. Por fim, serão apresentados os trabalhos relacionados e as considerações finais desse estudo.

\section{IMS Learning Design}

O IMS Learning Design (IMS LD) é um padrão aberto usado para representar uma grande variedade de estratégias pedagógicas de maneira formal, semântica, interoperável e legível por computador [Oliveira et al., 2014]. Esta especificação foi desenvolvida pelo IMS Global Learning Consortium ${ }^{1}$, uma organização internacional composta por renomadas universidades e empresas de tecnologia.

O padrão IMS LD é composto por um conjunto de especificações para organização de objetos de aprendizagem e uma linguagem para modelagem de sistemas educativos que relaciona pessoas (como alunos e professores), recursos e serviços de forma coordenada. A especificação é composta por elementos que, quando integrados para representar um processo de ensino e aprendizagem, constituem uma Unidade de Aprendizagem (em inglês Unit of Learning - UoL).

\footnotetext{
http://www.imsglobal.org/membersandaffiliates.html
} 
A linguagem do padrão IMS LD caracteriza-se como uma DSL baseada em XML para fornecer uma codificação interpretável por computador de processos de ensino e aprendizagem suportados por tecnologias digitais. Portanto, torna-se necessário o uso de ferramentas de autoria ou editores (como o Reload ${ }^{2}$ ) para que os professores possam especificar suas aulas em IMS LD. Para interpretação e execução de uma unidade de aprendizagem em IMS LD existem os programas conhecidos como players IMS LD, por exemplo, o CopperCore ${ }^{3}$.

\section{Contextualização da Pesquisa}

\subsection{Cenário}

Um dos principais objetivos de uma aula de campo é proporcionar aos alunos experiências práticas sobre o que eles aprenderam em sala de aula [Shakil et al., 2011]. Durante sua execução, os alunos experimentam no ambiente real o que aprenderam em sala de aula e são estimulados a realizar inúmeras atividades, que irão variar de acordo com a área estudada e o público alvo. No contexto desse trabalho, com o propósito de ilustrar um cenário para a abordagem proposta será utilizada uma aula de campo sobre Geologia. Para auxiliar a visualização desse cenário, foi elaborada a Figura 1, que ilustra uma aula de campo com professor e alunos utilizando smartphones. Para composição dessa imagem, tomamos como base as características do sistema móvel e ubíquo apresentado em [Marçal et al., 2014].

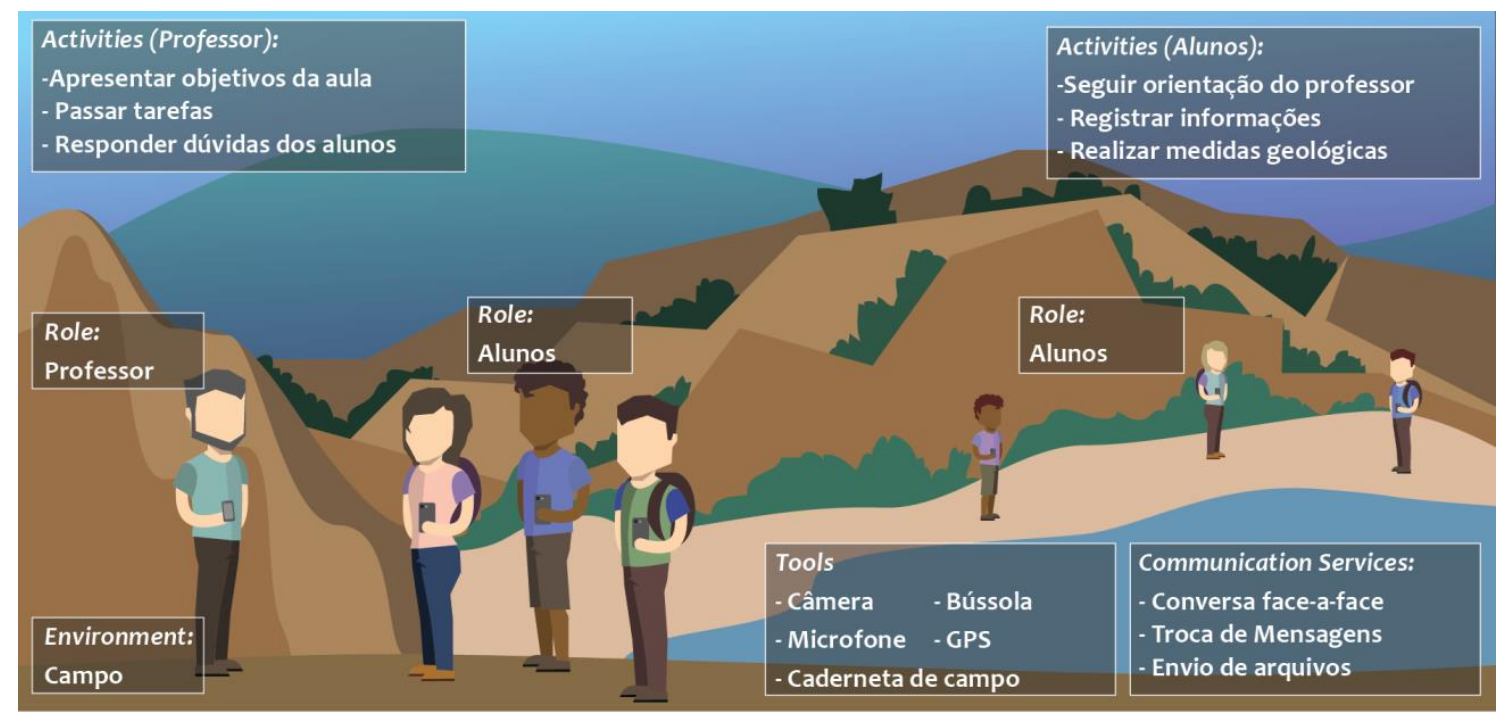

Figura 1. llustração de uma aula de campo com smartphones e elementos IMS LD.

As caixas de textos da Figura 1 exibem um mapeamento entre os elementos do ambiente real e aqueles presentes na linguagem IMS LD. O cenário ilustrado apresenta elementos que não precisam das tecnologias móveis e ubíquas, como a atividade "Apresentar objetivos da aula", e outros que precisam, como o serviço "Envio de arquivos".

Nesse cenário, existem dois elementos IMS LD do tipo $<$ role $>$ (professor e aluno), que representam os participantes da unidade de aprendizagem. Cada um deles

\footnotetext{
2 http://www.reload.ac.uk/ldeditor.html

3 http://coppercore.sourceforge.net/
} 
pode executar $<$ activities $>$, que equivalem às atividades de aprendizagem que os alunos podem executar e aquelas de suporte realizadas pelo professor. O local onde ocorre a aula de campo é codificado em IMS LD através do elemento <enviroment $>$, que nesse exemplo é um campo ${ }^{4}$. Entre os elementos que podem compor um <environment $>$, têmse: as ferramentas $(<$ tool-objects $>)$ disponíveis; e os serviços para a comunicação entre os participantes ( $<$ communication-services $>$ ).

\subsection{Limitações da IMS Learning Design}

IMS-LD é uma especificação para descrever processos de aprendizagem que pretende ser pedagogicamente neutra e capaz de expressar uma diversidade de situações de aprendizagem, em especial, aquelas mediadas por computador. Entretanto, deficiências já foram apontadas na literatura quanto a sua generalidade e a necessidade de extensões [Derntl et al, 2012; Burgos, 2012; De-la-Fuente-Valentín et al., 2011].

No caso das aulas de campo, um complicador à utilização da especificação IMS LD na forma original é a ausência de conexão com a Internet em diversos momentos no campo. Este fato dificulta o uso da IMS LD porque seus elementos foram desenvolvidos considerando alunos conectados ao ambiente virtual de aprendizagem. Por exemplo, no caso dos serviços de comunicação entre os participantes, a IMS LD prevê apenas os elementos <conference $>$ e <send-mail $>$, que não contemplam a especificação de serviços como a troca de arquivos e envio de mensagens utilizando tecnologias sem fio.

Outra limitação da IMS LD, com relação a sistemas ubíquos, diz respeito à ausência nesse padrão de elementos para especificação da captura de dados dos sensores dos dispositivos móveis, como câmera, microfone, GPS e acelerômetro. Um elemento que poderia ser utilizado para contornar essa limitação seria o <learning-object $>$. Porém, conforme [De-la-Fuente-Valentín et al., 2011], apesar de ampliar os recursos da UoL, o <learning-object $>$ não possibilita a integração com ferramentas externas.

Além das limitações exemplificadas, outros requisitos importantes para sistemas móveis e ubíquos voltados a aulas de campo não são previstos pelo IMS LD, tais como: a persistência local dos dados e o uso das informações contextuais ${ }^{5}$ do aluno em campo. Assim, percebe-se que extensões e adaptações são necessárias para possibilitar a modelagem desse tipo de sistema usando o padrão IMS LD.

\section{A Extensão ML4UL}

Esta seção descreve a ML4UL (Modeling Language for Ubiquitous Learning), uma extensão à linguagem IMS LD que tem como principal objetivo permitir a especificação de sistemas móveis e ubíquos voltados para aulas de campo. A premissa fundamental da ML4UL é a manutenção da estrutura da IMS LD. A Figura 2 apresenta os principais elementos da IMS LD (em cinza) e os propostos na ML4UL (em amarelo).

As alterações e adaptações propostas pela ML4UL não afetam o modelo padrão. Desta forma, pretende-se manter todos os benefícios da especificação original e adicionar novos com foco nas tecnologias móveis e ubíquas aplicadas a aulas de campo. As mudanças propostas são descritas nas próximas subseções.

\footnotetext{
${ }^{4}$ Campo é a terminologia adotada nas aulas de campo de geologia para indicar o local onde elas acontecem.

${ }^{5}$ Informação contextual é todo e qualquer dado que possa ser utilizado para caracterizar uma entidade (pessoa, local ou objeto) relevante para a interação entre o usuário e o sistema [Dey e Abowd, 1999].
} 


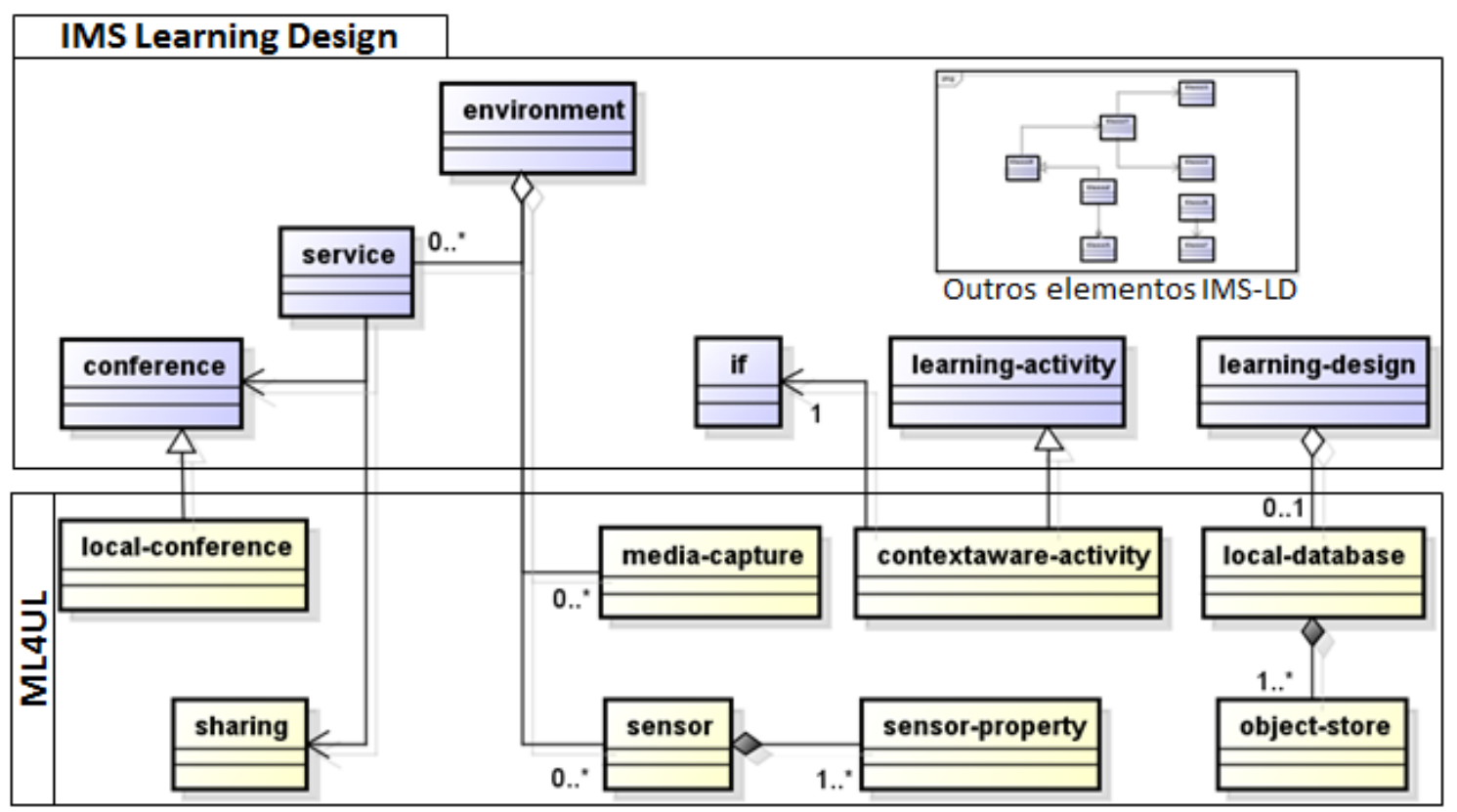

Figura 2. Visão geral dos novos elementos propostos pela ML4UL

\subsection{Novos Serviços de Comunicação}

Durante alguns momentos da aula de campo, os alunos estão dispersos geograficamente e precisam esclarecer dúvidas com o professor ou trocar informações com os colegas. As tecnologias móveis e ubíquas têm sido utilizadas para ampliar essa comunicação entre alunos e professores em campo, seja via chat textual [Chiang et al., 2014] ou via vídeo [Stefan et al., 2013].

Desta forma, propomos na ML4UL o elemento <local-conference> que é uma especialização do elemento <conference $>$ da IMS LD. Através desse novo elemento será possível especificar: a atividade de chat local, o tipo de tecnologia sem fio a ser utilizada (como Bluetooth ou Wi-Fi Direct), os parâmetros para essa conexão; e, de que forma se dará a comunicação entre os participantes (e.g. texto, áudio ou vídeo).

Um recurso para comunicação utilizado para sistemas de mobile learning são as mensagens instantâneas dos dispositivos móveis (serviço conhecido como Mobile Instant Messaging). Essas mensagens, diferentemente dos chats, não pressupõem uma sessão para interação entre todos os participantes. Outra importante ferramenta de interação entre participantes de uma aula de campo é a possibilidade da troca de arquivos (como fotos ou gravações). A IMS LD não dispõe de um elemento que permita a representação nem das mensagens instantâneas nem do compartilhamento de arquivos entre os participantes. Portanto, propomos como solução para essas limitações o elemento <sharing>. Através dele é possível especificar o compartilhamento de mensagens ou arquivos entre os envolvidos na aula de campo.

\subsection{Novas Ferramentas}

Um requisito importante presente em pesquisas sobre o uso dos sistemas móveis e ubíquos em aulas de campo é a captura de dados através de sensores [Marçal et al., 2013; Wu et al., 2013]. A obtenção dessas informações é importante para indicar o contexto em que o aluno se encontra e permitir que, a partir delas, decisões sejam 
tomadas. Considerando que a IMS LD não fornece suporte a modelagem desse tipo de recurso, propomos dois novos elementos para solucionar essa questão: <mediacapture $>\mathrm{e}<$ sensor $>$. Ambos teriam atributos para indicar as configurações dos sensores e para receber os dados capturados.

$\mathrm{O}<$ media-capture $>$ é o elemento responsável por permitir a especificação dos mecanismos de captura de imagens, áudios e vídeos. Alguns exemplos de atributos desse elemento são $<$ media-type $>$ e $<$ media-file $>$, que indicam respectivamente o tipo de mídia a ser obtida (imagem ou vídeo ou áudio) e o caminho do arquivo que armazenará a mídia capturada.

Através do elemento <sensor> é possível modelar o funcionamento dos sensores relacionados aos movimentos realizados pelos alunos em seus dispositivos móveis, à localização em que eles se encontram e às variáveis ambientais naquele instante, por exemplo: bússola, GPS e luminosidade. O elemento <sensor $>$ é composto de elementos <sensor-property>, que são usados para especificar as propriedades do sensor, como latitude e longitude no caso do GPS.

\subsection{Persistência Local dos Dados}

A IMS LD assume que as interações dos participantes entre si e com o ambiente virtual de aprendizagem ocorrem através da Internet. Porém, no caso das aulas de campo, em diversos momentos os alunos não têm conexão e precisariam interagir com o sistema móvel e ubíquo, por exemplo, para armazenar dados sobre o local em que se encontram. Através da IMS LD não é possível especificar essa funcionalidade.

Para solucionar essa questão da gerência dos dados locais, propomos na ML4UL uma solução baseada em dois elementos. $\mathrm{O}<$ objectstore $>$, que seria responsável por representar o armazenamento dos registros. Cada registro consiste de uma chave e um valor. O outro elemento proposto é o <local-database $>$, que consiste em um conjunto de $<$ objectstore $>$. Cada Unidade de Aprendizagem pode ter um $<$ local-database $>$ que pode conter um ou vários $<$ objectsore $>$.

Os principais motivos para escolha desta solução foram: esta proposta é baseada em uma especificação de armazenamento de dados local do W3C (World Wide Web Consortium), a Indexed Database $A P I^{6}$; a IndexedDB (como esta especificação é conhecida) é um padrão da HTML5 e consequentemente compatível com vários browsers web e sistemas operacionais de dispositivos móveis; por último, este padrão apresenta uma estrutura mais simples que em modelos de banco de dados relacionais.

\subsection{Sensibilidade ao Contexto}

Além de serviços de comunicação e recursos de sensoriamento, os sistemas móveis e ubíquos podem fazer uso das informações contextuais para ampliar os benefícios das aulas de campo. No caso da aula de geologia do cenário apresentado, um exemplo de informação de contexto importante é a localização do aluno. A partir dela, um sistema móvel e ubíquo pode, por exemplo, fornecer vídeos informativos e conteúdo didático sobre um determinado afloramento no qual o aluno se encontra ou notificar o aprendiz de uma atividade que precisa ser realizada naquele contexto.

\footnotetext{
${ }^{6}$ http://www.w3.org/TR/IndexedDB/
} 
Considerando as dificuldades para especificar atividades sensíveis ao contexto apontadas em [Burgos, 2012] e utilizando como base as soluções desenvolvidas por Gómez et al. (2014) e Mavroudi et al. (2012), propomos o elemento <contextawareactivity $>$. Este tem como objetivo facilitar a modelagem de atividades adaptativas e sensíveis ao contexto. Ele seria uma especialização do elemento <learning-activity>, porém com um atributo adicional e obrigatório: o <if $>$. Assim, a <contextawareactivity> só deve ser executada se as condições especificadas no seu elemento <if> são satisfeitas.

\section{Demonstração da Extensão}

Para demonstrar o funcionamento da ML4UL, utilizaremos como cenário de exemplo uma aula de campo de geologia, conforme ilustrada na Figura 1. Nesse exemplo, o aluno deve primeiramente utilizar o dispositivo móvel para visualizar as informações e os objetivos da aula. Posteriormente, ele deve anotar informações (via texto, áudio ou foto) sobre aquele ponto que está estudando. Em seguida, se ele estiver em uma determinada região, ele poderá assistir a um vídeo sobre o afloramento em que ele se encontra localizado. Por fim, o aluno pode anotar mais observações e depois deve transmitir em campo as anotações ao professor.

A Figura 3 mostra um diagrama de atividades da UML que representa graficamente a aula utilizada como exemplo e trechos da especificação em IMS LD com a extensão ML4UL. Na codificação, os elementos da IMS LD e da ML4UL têm como prefixos os namespaces imsld e $m l 4 u l$, respectivamente. As linhas de código relativas à ML4UL estão em azul e vermelho. Tendo em vista o fato das especificações em IMS LD serem muito extensas e devido à limitação de páginas do artigo, são apresentados os códigos de apenas dois elementos da ML4UL: <sensor $>$ e <contextaware-activity $>$. Porém, para representar a aula do exemplo também são necessários os seguintes outros elementos: <media-capture > para indicar a captura de foto e áudio; e, <sharing > para especificar o compartilhamento em campo das informações anotadas.

O primeiro trecho de codificação exemplifica a especificação do elemento $<$ sensor> da ML4UL. São mostrados: o atributo "sensing-type", que indica o tipo de sensor a ser usado; e o elemento <sensor-property>, usado para indicar quais as propriedades deverão receber os valores de retorno após a execução do sensor. Considerando esse código, o programa interpretador de IMS LD com ML4UL deverá chamar o sensor de geolocalização, obter as coordenadas e atualizar os valores nas propriedades especificadas.

O segundo código extraído mostra o elemento da ML4UL < contextawareactivity $>$. Nele, além dos elementos padrões do <learning-activity> da IMS LD, como $<$ activity-description $\rangle$ e $\langle$ title $\rangle$, aparece o elemento $<$ if $>$. Na condição mostrada nesse exemplo, é verificado se a propriedade "Localizacao_aluno", obtida através do elemento <sensor>, está em uma determinada localização, representada pela propriedade "Placel". 


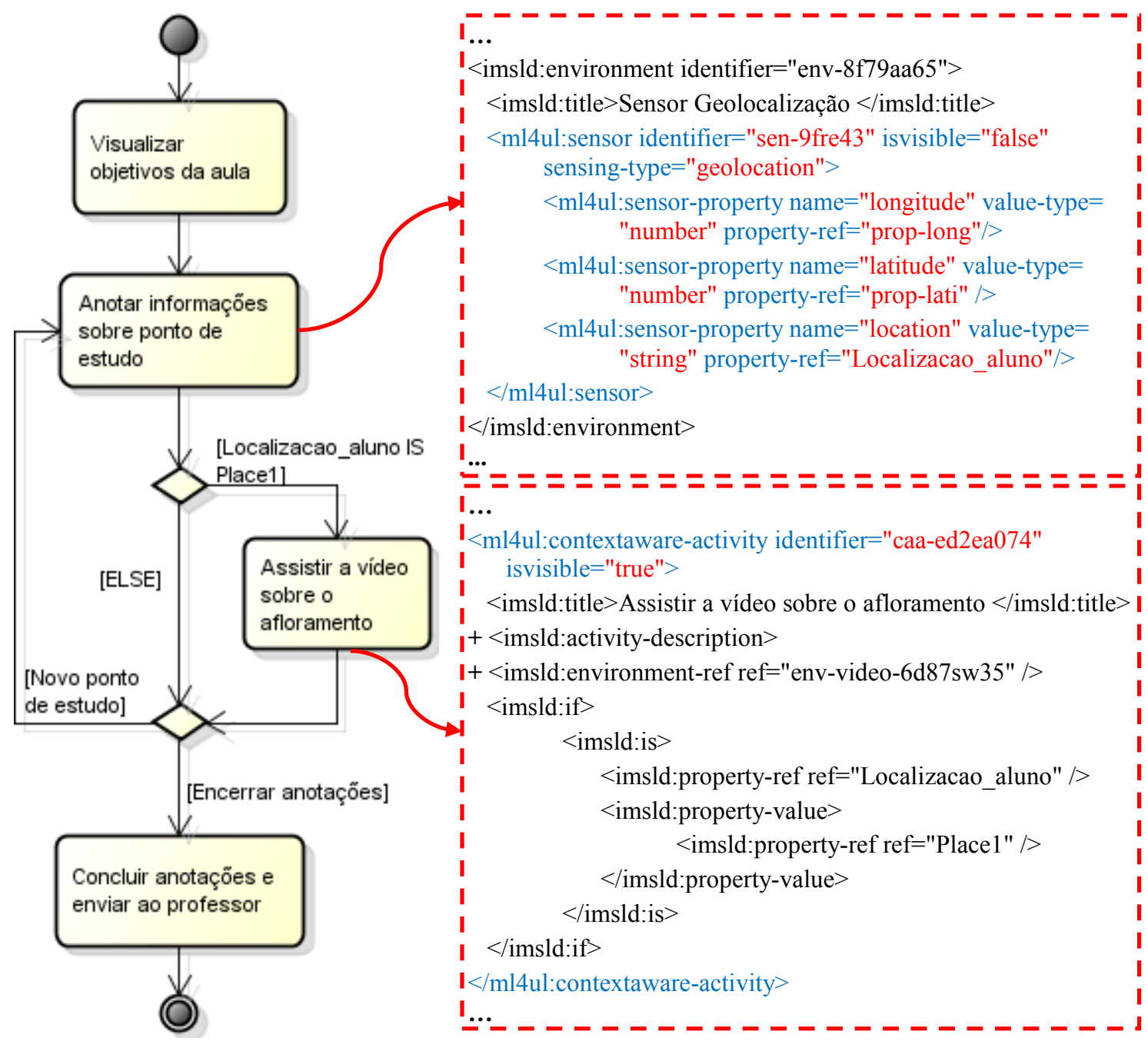

Figura 3. Exemplo em Diagrama de Atividades UML e codificação em IMS LD com ML4UL

Os trechos de código demonstram a conformidade da extensão com o padrão IMS LD e como devem ser especificados alguns dos elementos propostos na ML4UL. A partir dos exemplos, também é possível constatar que alguns editores existentes de IMS LD podem ser usados para especificar os novos elementos. Por fim, os trechos de codificação permitem uma análise inicial sobre qual seria o esforço para um programa interpretar essa especificação e executá-la diretamente no dispositivo móvel ou qual seria a dificuldade de conversão para um aplicativo móvel.

\section{Trabalhos Relacionados}

Em [Gómez et al., 2014], os autores apresentam uma abordagem para especificação de unidades de aprendizagem adaptativas e sensíveis ao contexto para dispositivos móveis baseada na IMS LD. De forma semelhante, o estudo apresentado em [Hebiri et al., 2013] mostra uma solução que combina padrões, como o IMS LD, para possibilitar a especificação de unidades de aprendizagem para dispositivos móveis com foco em pessoas com deficiência. Nesses estudos, o foco está na questão da adaptação do conteúdo, porém não é prevista a representação de recursos importantes em aulas de campo suportadas por dispositivos móveis, tais como: sensores, persistência local dos dados e ferramentas de comunicação via rede sem fio. 
Outra abordagem consiste na modelagem de sistemas móveis e ubíquos através de linguagens mais focadas na tecnologia. Por exemplo, em [Salman e Pourabdollah, 2010] é apresentada uma solução baseada em XML para codificação de elementos de sensoriamento em sistemas, tais como: sensor, para representação dos sensores; e, response, que indica a ação a ser executada pelo dispositivo móvel após a captura dos dados pelo sensor. Porém, esta solução está focada nos aspectos tecnológicos e não considera elementos importantes em práticas educativas, como o fluxo da aprendizagem e o perfil dos participantes.

A linguagem proposta neste artigo, ML4UL, se difere dos trabalhos apresentados em dois sentidos: por um lado ela prevê elementos para representação de recursos importantes em sistemas móveis e ubíquos para aulas de campo, e por outro lado, utiliza como base um padrão internacional de modelagem de práticas educativas aceito por diversas instituições de ensino. Então, a ML4UL se propõe a contemplar em uma única abordagem os aspectos tecnológicos e os pedagógicos, diferentemente dos trabalhos relacionados que focam em um dos dois aspectos de forma individualizada.

\section{Conclusão}

O padrão IMS LD possui uma estrutura flexível e personalizável que permite a modelagem de diferentes processos de ensino e aprendizagem. Entretanto, ao se considerar as aulas de campo suportadas por sistemas móveis e ubíquos, recursos importantes não são contemplados pelo padrão. Para solucionar essa questão, apresentamos nesse artigo a ML4UL, uma extensão ao IMS LD que possibilita a modelagem de aulas de campo apoiadas por sistemas móveis e ubíquos.

A ML4UL se propõe a ampliar as possibilidades de modelagem da IMS LD ao incluir elementos para especificação de recursos como: sensores (por exemplo, GPS, bússola e câmera), interação entre os participantes via redes sem fio, persistência local de dados e atividades de aprendizagem adaptativas e sensíveis ao contexto. A principal contribuição da ML4UL está no fato de possibilitar a modelagem de sistemas móveis e ubíquos voltados para aulas de campo, tendo como base um padrão de especificação de práticas educativas reconhecido internacionalmente.

Como pesquisas futuras, pretendemos trabalhar em duas linhas: desenvolver um software editor que permita a modelagem gráfica das aulas de campo usando a ML4UL; e, possibilitar a execução no dispositivo móvel das aulas especificadas usando a extensão. Para isso, será construído um aplicativo que interpretará a aula em ML4UL no próprio dispositivo do aluno ou um sistema que converterá a especificação para um aplicativo executável no sistema operacional alvo.

\section{Referências}

Brambilla, M., Cabot, J., e Wimmer, M. (2012). Model-driven software engineering in practice. Synthesis Lectures on Software Engineering, 1(1), 1-182.

Burgos, D. (2012). How to use IMS LD to support eLearning in an effective way (invited talk). In International Symposium on Computers in Education (SIIE), 2012.

Chiang, T. H., Yang, S. J., e Hwang, G. J. (2014). Students' online interactive patterns in augmented reality-based inquiry activities. Computers e Education, 78, 97-108. 
Dey, A. K. e Abowd, G. D. (1999). Towards a better understanding of context and context-awareness. In: GELLERSEN, H.-W. (Ed.). Handheld and Ubiquitous Computing. [S.1.]: Springer Berlin Heidelberg, 1999, p. 304-307.

Derntl, M., Neumann, S., Griffiths, D., e Oberhuemer, P. (2012). The conceptual structure of IMS Learning Design does not impede its use for authoring. Learning Technologies, IEEE Transactions on, 5(1), 74-86.

De-la-Fuente-Valentín, L., Pardo, A., e Kloos, C. D. (2011). Generic service integration in adaptive learning experiences using IMS learning design. Computers \& Education, 57(1), 1160-1170.

Gómez, S., Zervas, P., Sampson, D. G., e Fabregat, R. (2014). Context-aware adaptive and personalized mobile learning delivery supported by UoLmP. Journal of King Saud University-Computer and Information Sciences, 26(1), 47-61.

Hebiri, M. H., Jemni, B.A. L., Jemni, M., e Sampson, D. G. (2013). Towards accessible and personalized mobile learning for learners with disabilities. In IEEE Fourth International Conference ICTA 2013 (pp. 1-6).

Koper, R., e Miao, Y. (2007). Using the IMS LD standard to describe learning designs. Disponível em http://nx-hrl-075v.web.pwo.ou.nl/handle/1820/927. Último acesso em 15 de maio de 2015.

Marçal, E., Andrade, R. M., Viana, W., Rodrigues, D., Maia, M., Mendes, W., Freire J. e Benicio, W. (2013). Geomóvel: Um Aplicativo para Auxílio a Aulas de Campo de Geologia. In Anais do SBIE 2013 Simpósio Brasileiro de Informática na Educação.

Marçal, E., Viana, W., Andrade, R., e Rodrigues, D. (2014). A mobile learning system to enhance field trips in geology. In IEEE Frontiers in Education Conference (FIE), 2014 (pp. 1-8).

Mavroudi, A., e Hadzilacos, T. (2012). Implementation of adaptive learning designs. Annals of the University of Craiova, Series: Automation, Computers, Electronics and Mechatronics, 9(2).

Oliveira, F. H., Nascimento Salvador, L., e Novais, R. (2014). Uma Análise do Uso da Ontologia IMS LD na Construção de Modelos Conceituais para E-learning. In Anais do SBIE 2014 Simpósio Brasileiro de Informática na Educação.

Salman, A. M., e Pourabdollah, A. (2010). UCML: Ubiquitous Control Modeling Language for Android Platform. In IEEE NGMAST 2010 (pp. 112-116).

Shakil A., Faizi W. e Hafeez S. (2011) The need and importance of field trips at higher level in karachi, pakistan. In: International Journal of Academic Research in Business and Social Sciences, June (2011) Vol. 2, No. 1.

Sharples, M. e Roschelle, J. 2010. "Guest Editorial - Special section on Mobile and Ubiquitous Technologies for Learning." IEEE Transactions on Learning Technologies, Vol. 3, no. 1, pp. 4-6.

Stefan, L., Gheorghiu, D., Moldoveanu, F., e Moldoveanu, A. (2013). Ubiquitous Learning Solutions for Remote Communities--A Case Study for K-12 Classes in a Romanian Village. In IEEE CSCS 2013 19th (pp. 569-574).

Wu, P. H., Hwang, G. J., e Tsai, W. H. (2013). An Expert System-based Context-Aware Ubiquitous Learning Approach for Conducting Science Learning Activities. Educational Technology e Society, 16(4), 217-230. 